\title{
The prevalence and awareness of sleep apnea in patients suffering chronic pain: an assessment using the STOP-Bang sleep apnea questionnaire
}

This article was published in the following Dove Press journal:

Nature and Science of Sleep

\section{Gregory S Tentindo' \\ Scott M Fishman ${ }^{2}$ \\ Chin-Shang $\mathrm{Li}^{3}$ \\ Qinlu Wang ${ }^{4}$ \\ Steven D Brass ${ }^{5,6}$}

'Department of Oral and Maxillofacial Surgery, University of California San Francisco, San Francisco, CA, USA;

${ }^{2}$ Department of Anesthesiology, Division of Pain Medicine, University of California Davis, Sacramento, CA, USA; ${ }^{3}$ Department of Public Health Sciences, Division of Biostatistics, University of California Davis, Sacramento, CA, USA; ${ }^{4}$ Department of Statistics, University of California Davis, Davis, CA, USA; ${ }^{5}$ Department of Neurology, University of California Davis, Sacramento, CA, USA;

${ }^{6}$ Department of Administration, Providence Little Company of Mary Medical Center San Pedro, San Pedro, CA, USA

Correspondence: Steven D Brass Providence Little Company of Mary Medical Center San Pedro, I300 W. 7th Street, San Pedro, CA 90732, USA

$\mathrm{Tel}+\mathrm{I} 3105145494$

Email steven.brass@providence.org
Purpose: Some patient subsets are at higher risk of sleep apnea, including patients with chronic pain. However, it is unclear whether patients and their caregivers are aware of the possibly increased risk of sleep apnea in this population. Chronic pain is often treated with opioids which may decrease both the central respiratory drive and the patency of the upper airway, potentially contributing to this sleep disorder. Using a self-reporting questionnaire approach in the chronic pain population, this study surveyed patient and caregiver awareness surrounding the risk of sleep apnea. In addition, we looked at the influence of opioid therapy on the prevalence of sleep apnea. Participants and methods: Consecutive patients presenting to a pain clinic were invited to participate anonymously in a survey that included the STOP-Bang sleep apnea questionnaire, which assesses patients' knowledge, testing, diagnosis, or treatment of sleep apnea and whether their caregivers had discussed with them their increased risk of sleep apnea and opioid use. Results: Among 305 participating patients, 58.2\% $(\mathrm{n}=173)$ screened positive for sleep apnea. Among the 202 patients on opioid therapy, 59.2\% (116/202) were STOP-Bang positive (score $\geq 3$ ). However, only $37.5 \%$ ( $n=72 / 173)$ of these patients had discussed their risk of sleep apnea with a caregiver and only $30.7 \%(n=59)$ underwent testing. Against expectation, opioids did not increase the prevalence of sleep apnea in our study population.

Conclusion: Chronic pain patients had a high risk of sleep apnea, regardless of opioid prescription. Most patients were unaware of their increased risk and denied undergoing the necessary testing. Greater attention to screening, testing, and education for sleep apnea needs to be paid in chronic pain patients, especially given the potentially dangerous ramifications of opioidinduced sleep apnea.

Keywords: sleep apnea, opioids, narcotics, STOP-Bang survey

\section{Introduction}

An estimated 100 million Americans suffer from chronic pain disorders. ${ }^{1}$ This number includes patients with cancer and non-cancer pain. In the latter group, chronic noncancer pain (CNCP) patients can suffer from a wide variety of painful conditions such as fibromyalgia, arthritis, and other inflammatory conditions, afflicting primarily an older demographic.

Sleep apnea affects $2 \%-14 \%$ in community-screened normal populations, but has a much higher prevalence in certain patient subgroups. ${ }^{2}$ Twenty to $90 \%$ of individuals referred for sleep studies, a physician-selected cohort, are affected by sleep apnea. ${ }^{2,3}$ Males are three times more likely to experience sleep apnea. ${ }^{4}$ Further, the prevalence 
increases with age, especially in people more than 60 years old, and with obesity. ${ }^{3,4}$

Sleep-disordered breathing has two major manifestations, central sleep apnea (CSA) or obstructive sleep apnea (OSA). Hence, sleep apnea may be a consequence of central depression of respiratory rate, amplitude, reflex responses, and reduced brain arousability, as well as the function of chemoreceptors that monitor changes in blood oxygen and carbon dioxide levels. ${ }^{5}$ Alternatively, sleep apnea is due to obstruction by soft tissues of the upper airway. ${ }^{6}$ Sleepdisordered breathing is associated with an increased risk for cardiovascular and cerebrovascular disease, as well as a multitude of other morbidities. Patients with untreated sleep-disordered breathing have a higher mortality risk, independent of age, gender, and body mass index (BMI). ${ }^{7}$

Pain causes sleep disorders such as wakefulness and sleep apnea. There is a relationship between pain and sleep disturbances. ${ }^{8,9}$ Over $70 \%$ of patients with chronic pain disorders report sleep disruption, and lack of restful sleep increases hyperalgesia. ${ }^{89}$ By contrast, sleep rebound, especially in non-REM sleep may have an analgesic effect. ${ }^{9}$ Hence, pain induces sleep disorders and lack of sleep increases pain.

Opioids are widely used to treat chronic pain. In 1986 Portenoy and Foley published a review of 38 cases of CNCP patients successfully treated with opioids, ${ }^{10}$ leading to wider use in the management of CNCP. Today, it is ethically mandated to treat $\mathrm{CNCP},{ }^{11}$ resulting in a four-fold increase in opioid prescription during the 2000-2010 period. Unfortunately, accidental opioid overdose, death, and dependency have increased dramatically, ${ }^{12}$ reaching "epidemic" proportions ${ }^{13,14}$ and prescription opioids for chronic pain are recognized as one of the roots of this problem. ${ }^{15,16}$

Opioid medications cause numerous unwanted and adverse effects, apart from overdose. Of note, opioids have been shown to decrease both central respiratory drive and the patency of the upper airway. Hence, sleep-disordered breathing is observed in patients on chronic opioid therapy. An observational study of chronic pain patients on opioid therapy who received overnight polysomnograms demonstrated an abnormal apnea-hypopnea index ( $\geq 5$ per hour) in $75 \%$ of patients, with a mixture of both central and obstructive apneas. ${ }^{8} \mathrm{~A}$ dose-dependent relationship between chronic opioid use and the development of a peculiar pattern of respiration, consisting of central apneas and ataxic breathing, has also been shown. ${ }^{17}$ Patients receiving sustainedrelease opioid medications have respiratory abnormalities which are unique compared to opioid-free patients with OSA. Longer, more severe hypoxia was observed in NREM compared to REM sleep. During NREM sleep, irregular respiratory pauses and gasping without periodicity were present. Nasal positive airway pressure (CPAP) was shown to be ineffective in these patients. ${ }^{18}$

Proper identification of chronic pain patients at a higher risk for sleep-disordered breathing may be a difficult task to accomplish due to the sedative effects of opioid medications, which overlap with vague symptoms of sleep apnea. Screening questionnaires have been developed to identify individuals at a higher risk for sleep apnea, including the checklist of the American Society of Anesthesiology, the STOP questionnaire (snoring, tiredness, observed apnea, and high blood pressure), the STOP-Bang questionnaire (STOP plus BMI, age, neck circumference, gender), and the Epworth Sleepiness Scale. The STOP-Bang questionnaire is a concise and easy-to-use, eight point, dichotomized (yes/ no) screening tool. ${ }^{19}$ In a systematic review of screening questionnaires for sleep apnea, the STOP-Bang questionnaire had the highest methodological quality and sensitivity. ${ }^{20}$ It has been validated in different populations, including surgical patients, ${ }^{21}$ hospitalized patients, ${ }^{22}$ and patients presenting to a sleep disorders unit. ${ }^{23}$

The medical literature is limited in terms of the methods used for the screening of patients on chronic opioids for sleep apnea. Chronic opioid users' awareness of their increased risk for sleep apnea is also unknown. It is important to identify those chronic pain patients who have sleep apnea, so that early treatment can be initiated in order to improve their quality of life and health status. The aims of this study were to: 1) investigate risk of sleep apnea in the chronic pain population, 2) assess knowledge and awareness of risk for sleep apnea in patients, 3) evaluate the discussion and assessment of sleep-disordered breathing with physicians as a measure of their awareness, and 4) determine whether the use of opioids increases the prevalence of sleep apnea in the chronic pain population. This study was also intended to bring to the attention of the sleep and pain medicine communities, as well as general medical practitioners, the extent to which sleep apnea may be under-diagnosed and under-treated in the chronic pain population.

\section{Participants and methods Participants}

The University of California Davis Pain Management Clinic provides cutting-edge treatment of significant chronic pain through a multidisciplinary approach. The clinical team includes anesthesiologists, internists, and psychiatrists with expertise in pain management to restore function and decrease pain. 
The study protocol was approved by the UC Davis Institutional Review Board (IRB number 637877-1). The study population was recruited from 357 consecutive patients presenting for their scheduled consultation at the Pain Management Clinic between September 9 and December 23, 2014. Patients were invited to participate in an anonymous survey. Subjects were excluded from the study if they declined to participate or were under 18 years of age. Of the 357 patients who were invited to participate, 305 met the inclusion criteria. Most of the patients attending this specialist consultation clinic who were on opioid therapy received their prescriptions from external community providers, such as their primary care physicians.

\section{Procedure}

All survey and questionnaire data collected were self-reported by patients and considered anonymous. Thus, confirmation of the disease status and medication relied on self-report. Patients were asked to complete the survey on their own, while waiting for their physician in treatment rooms.

\section{Measures}

The survey comprised eight pages and included diagnosis of pain disorder (yes, no), consumption of narcotic pain medication including type and duration, and a detailed sleep history (Supplementary material). The survey also included standardized validated questionnaires related to sleepiness and sleep-disordered breathing. We also noted whether patients reported having knowledge of sleep apnea, whether they had discussed sleep apnea with a health care professional, and if they had been tested for, diagnosed with or treated for sleep apnea. Completion took approximately 5 minutes.

The STOP-Bang questionnaire, described in detail elsewhere (http://www.stopbang.ca/osa/screening.php) is comprised of eight yes/no questions relating to the risk factors for OSA. Questions include snoring, daytime tiredness, observed apneas, high blood pressure, BMI (greater than $35 \mathrm{~kg} / \mathrm{m}^{2}$ ), age (greater than or equal to 50 years), neck circumference (greater than $40 \mathrm{~cm}$ ), and gender (male). Measurement of height and weight, and the online calculation of BMI, were performed by the registered nurses as part of the regular medical visit. A tape measure was included in the survey, with instructions and anatomical guidelines for measuring neck circumference. A trained research assistant was present to assist with the neck circumference measurement. A patient was considered at risk for OSA if they had three or more positive responses on the STOPBang questionnaire. ${ }^{19}$
The Epworth Sleepiness Scale (http://epworthsleepinessscale.com/about-the-ess/) is used to measure daytime sleepiness by assessing the patient's likelihood of falling asleep under eight common scenarios. Likelihood is scored between 0 (never) and 3 (high chance) with a total score out of 24. A score greater than 10 is indicative of excessive daytime sleepiness. ${ }^{24}$

\section{Statistical analysis}

Chi-squared test was used to study the association between two categorical variables. A $P$-value $<0.05$ was considered statistically significant. All analyses were performed with SAS v9.4 (SAS Institute Inc., Cary, NC, USA).

\section{Results}

Our study population had a mean age of $57.5+13.9$ years. The majority were female (66.1\%) and Caucasian $(81.2 \%)$ (Table 1$)$. The majority of the participants $(72.2 \%)$ were overweight or obese (BMI $\geq 25)$ (Table 1). Ninety-one point nine percent $(n=274)$ of the entire cohort was diagnosed with a pain disorder of 6 months or longer. There were 202 (66.5\%) patients on narcotic pain medication. Of the patients who reported on their length of narcotic use, $75.7 \%(n=131)$ of them were using narcotic pain medication for 6 months or longer.

Table 2 reports on the prevalence of sleep apnea in pain patients, and their awareness of it. According to the STOPBang assessment, overall $58.3 \%$ were positive (score $\geq 3$ ) for sleep apnea. There was no difference in prevalence between opioid-treated and untreated patients. Most patients (90.7\%) were knowledgeable about sleep apnea, and this percentage was not different in the opioid and non-opioid subgroups. However, only approximately a third (35.9\%) of all patients had discussed their risk of sleep apnea with a health care provider, and this was true even in the patients using opioids who are at a theoretically greater risk. Furthermore, only $29.0 \%$ had ever been tested for sleep apnea and only $22.7 \%$ of the whole of our chronic pain population had been treated.

A summary of outcomes for the Epworth Sleepiness Scale and the STOP-Bang score screening questionnaires is reported in Table 3. Among all subjects, 24.2\% suffered excessive daytime sleepiness (Epworth Sleepiness Scale $>10)$. Among opioid users, $25.9 \%(\mathrm{n}=50)$ had an Epworth Sleepiness Scale score greater than 10, compared with $22.2 \%(\mathrm{n}=22)$ of the opioid non-users, a difference that was not significant $(P=0.58)$. According to the STOP-Bang score, $58.3 \%$ were "positive" for sleep apnea with a score of $\geq 3$. However, there was no significant difference $(P=0.82)$ 
Table I Demographic characteristics of opioid users and non-users

\begin{tabular}{|c|c|c|c|}
\hline Demographic characteristics & Opioid users $n=196$ & Non-opioid users $n=101$ & Total $n=297$ \\
\hline \multicolumn{4}{|l|}{ Age (years) } \\
\hline Mean \pm SD & $56.6 \pm 14.0$ & $59.2 \pm 13.6$ & $57.5 \pm 13.9$ \\
\hline \multirow[t]{2}{*}{ (median, range) } & $(57,19-90)$ & $(60,22-87)$ & $(58,19-90)$ \\
\hline & & $\mathrm{P}=\mathrm{NS}$ & \\
\hline \multicolumn{4}{|l|}{ Gender } \\
\hline Total & $193^{*}$ & $99 *$ & $292 *$ \\
\hline Female & I33 (68.9\%) & $60(60.6 \%)$ & $193(66.1 \%)$ \\
\hline Male & $60(31.1 \%)$ & $39(39.4 \%)$ & $99(33.9 \%)$ \\
\hline \multirow[t]{2}{*}{ Not known } & $3(1.6 \%)$ & $2(3.0 \%)$ & $5(1.7 \%)$ \\
\hline & & $P=N S$ & \\
\hline \multicolumn{4}{|l|}{ Race } \\
\hline Total & $188^{*}$ & $94 *$ & $282^{*}$ \\
\hline Caucasian & I 48 (78.7\%) & 81 (86.2\%) & $229(81.2 \%)$ \\
\hline African American & $13(6.9 \%)$ & $2(2.1 \%)$ & $15(5.3 \%)$ \\
\hline Asian & $5(2.7 \%)$ & $4(4.3 \%)$ & $9(3.2 \%)$ \\
\hline Hispanic & 17 (9.0\%) & $4(4.3 \%)$ & 21 (7.5\%) \\
\hline Native Hawaiian or Pacific Islander & I (0.5\%) & $0(0.0 \%)$ & I (0.4\%) \\
\hline American Indian or Alaskan Native & $2(1.1 \%)$ & $0(0.0 \%)$ & $2(0.7 \%)$ \\
\hline Prefer not to answer & $2(1.1 \%)$ & $3(3.2 \%)$ & $5(1.8 \%)$ \\
\hline \multirow[t]{2}{*}{ Not known } & $8(4.3 \%)$ & $8(8.5 \%)$ & $15(5.3 \%)$ \\
\hline & & $P=N S$ & \\
\hline \multicolumn{4}{|l|}{ BMI } \\
\hline Total & $192 *$ & $99 *$ & $29 I^{*}$ \\
\hline Underweight $(\mathrm{BMI}<18.5)$ & $3(1.6 \%)$ & $2(2.0 \%)$ & $5(1.7 \%)$ \\
\hline Healthy weight $(\mathrm{BMI} \geq 18.5$ and $<25)$ & $48(25.0 \%)$ & $28(28.3 \%)$ & $76(26.1 \%)$ \\
\hline Overweight (BMI $\geq 25$ and $<30$ ) & $67(34.9 \%)$ & $33(33.3 \%)$ & $100(34.4 \%)$ \\
\hline Obese $(\mathrm{BMI} \geq 30)$ & $67(38.5 \%)$ & $36(36.4 \%)$ & $110(37.8 \%)$ \\
\hline \multirow[t]{2}{*}{ Not known } & $4(2.1 \%)$ & $3(3.0 \%)$ & $6(2.1 \%)$ \\
\hline & & $\mathrm{P}=\mathrm{NS}$ & \\
\hline \multicolumn{4}{|c|}{ Diagnosed or treated for pain disorder of $\geq 6$ months } \\
\hline Total & 196 & 101 & 297 \\
\hline Yes & 187 (95.4\%) & $87(86.1 \%)$ & 274 (91.9\%) \\
\hline \multirow[t]{2}{*}{ No } & $9(4.6 \%)$ & 15 (14.9\%) & $24(8.1 \%)$ \\
\hline & & $P=0.002$ & \\
\hline
\end{tabular}

Notes: Not all participants answered all questions. The number of respondents $(*)$ in each section are shown. Missing answers are given as "not known" in the table. $P$, significance of difference between opioid and non-opioid users.

Abbreviation: NS, not significant.

between the STOP-Bang score of $\geq 3$ in opioid users (59.2\%) and non-users $(56.4 \%)$. In fact, there was no difference in STOP-Bang score at any level between opioid users and non-users $(P=0.84)$.

Figure 1 depicts the use of different opioid medications amongst our study population. The majority of our subjects were either taking hydrocodone $(39.8 \%, \mathrm{n}=121)$ or oxycodone $(20.1 \%, \mathrm{n}=61)$. The doses being taken were not recorded because of concern that patients' recollection may be unreliable. Concomitant use of hypnotics was reported in $24.7 \%(n=50)$ of opioid users, compared to $14.6 \%(n=14)$ of opioid non-users.

Some of the patients were taking hypnotic medications. The survey asked about use of hypnotic medications, but did not request the dose. Of the opioid users, $24.7 \%$ were taking a hypnotic while $14.6 \%$ of non-users were, too. This difference did achieve minimal statistical significance $(P=0.046)$ but the frequency of OSA, according to the STOP-Bang score, did not differ in the patient groups using opioids vs those using opioids plus hypnotics.

\section{Discussion}

This survey found that a significant percentage of patients with CNCP are at risk for OSA. The proportion of CNCP patients screening positive on the STOP-Bang questionnaire for OSA is much higher than the prevalence reported in the general population. Given the relationship between chronic pain disorders and sleep disruption, ${ }^{8,9}$ it is understandable why almost $60 \%$ of our cohort screened at risk for sleep apnea using the STOP-Bang questionnaire. Remarkably, given the elevated risk associated with chronic pain, only approximately $35 \%$ of patients had discussed their apnea risk 
Table 2 Patient awareness of sleep apnea risks

\begin{tabular}{|c|c|c|c|}
\hline & Opioid users & Opioid non-users & Total \\
\hline STOP-Bang (score $\geq 3$ ) & $n=196$ & $n=101$ & $\mathrm{n}=297$ \\
\hline Positive & $116(59.2 \%)$ & $57(56.4 \%)$ & $173(58.3 \%)$ \\
\hline Negative & $80(40.8 \%)$ & $44(43.6 \%)$ & $124(4 \mid .7 \%)$ \\
\hline Do you know what sleep apnea is? & $\mathrm{n}=191 *$ & $\mathrm{n}=99 *$ & $\mathrm{n}=290^{*}$ \\
\hline Yes & $174(91.1 \%)$ & 89 (89.9\%) & $263(90.7 \%)$ \\
\hline No & $17(8.9 \%)$ & $10(10.0 \%)$ & $27(9.3 \%)$ \\
\hline Have you or your health care provider ever discussed your risk of sleep apnea? & $\mathrm{n}=192 *$ & $\mathrm{n}=98^{*}$ & $\mathrm{n}=290^{*}$ \\
\hline Yes & $72(37.5 \%)$ & $32(32.6 \%)$ & $104(35.9 \%)$ \\
\hline No & $120(62.5 \%)$ & $66(67.4 \%)$ & $186(64.1 \%)$ \\
\hline Have you ever been tested for sleep apnea? & $\mathrm{n}=192 *$ & $\mathrm{n}=98 *$ & $\mathrm{n}=290 *$ \\
\hline Yes & $59(30.7 \%)$ & $25(25.8 \%)$ & $84(29.0 \%)$ \\
\hline No & $133(69.3 \%)$ & $73(74.5 \%)$ & $206(71.0 \%)$ \\
\hline Have you ever undergone a sleep study (polysomnogram)? & $\mathrm{n}=192 *$ & $\mathrm{n}=99 *$ & $\mathrm{n}=291 *$ \\
\hline Yes & $62(32.3 \%)$ & $27(27.3 \%)$ & $89(30.6 \%)$ \\
\hline No & $130(67.7 \%)$ & $72(72.7 \%)$ & $202(69.4 \%)$ \\
\hline Have you ever been diagnosed with sleep apnea? & $\mathrm{n}=192 *$ & $\mathrm{n}=99 *$ & $\mathrm{n}=291 *$ \\
\hline Yes & $42(21.9 \%)$ & $24(24.2 \%)$ & $66(22.7 \%)$ \\
\hline No & $150(78.1 \%)$ & 75 (75.8\%) & $225(77.3 \%)$ \\
\hline Have you ever been treated with positive airway pressure (CPAP or BiPAP)? & $\mathrm{n}=192 *$ & $\mathrm{n}=97^{*}$ & $\mathrm{n}=289 *$ \\
\hline Yes & $42(21.9 \%)$ & $19(19.6 \%)$ & $61(21.1 \%)$ \\
\hline No & $150(78.1 \%)$ & $78(80.4 \%)$ & $228(78.9 \%)$ \\
\hline
\end{tabular}

Notes: None of the differences between groups were statistically significant by Chi-squared analysis. P-values ranged from 0.35 to 0.74 . Not all participants answered all questions. The number of respondents $(*)$ to each question is given.

Table 3 Summary of outcomes for Epworth and STOP-Bang screening questionnaires

\begin{tabular}{llll}
\hline Screening questionnaire & Opioid user group n=196 & Opioid non-user group n=10I & Total $\mathbf{n = 2 9 7}$ \\
\hline Epworth Sleepiness Scale score - median (range) & $7(0-22) \mathrm{n}=192 *$ & $7(0-23) \mathrm{n}=99 *$ & $7(0-23) \mathrm{n}=\left.29\right|^{*}$ \\
Epworth Scale score $>10$ (excessive daytime sleepiness) & $50 / 192 *(25.9 \%)$ & $22 / 99 *(22.2 \%)$ & $72 / 29 \mid *(24.2 \%)$ \\
STOP-Bang score 0 & $3(1.5 \%)$ & $0(0.0 \%)$ & $3(1.0 \%)$ \\
STOP-Bang score I & $24(12.3 \%)$ & $13(12.9 \%)$ & $37(12.5 \%)$ \\
STOP-Bang score 2 & $53(27.0 \%)$ & $31(30.7 \%)$ & $84(28.3 \%)$ \\
STOP-Bang score 3 & $49(25.0 \%)$ & $17(16.8 \%)$ & $66(22.2 \%)$ \\
STOP-Bang score 4 & $29(14.8 \%)$ & $22(21.8 \%)$ & $51(17.2 \%)$ \\
STOP-Bang score 5 & $19(9.7 \%)$ & $6(5.9 \%)$ & $25(8.4 \%)$ \\
STOP-Bang score 6 & $14(7.1 \%)$ & $8(7.9 \%)$ & $22(7.4 \%)$ \\
STOP-Bang score 7 & $4(2.0 \%)$ & $4(4.0 \%)$ & $8(2.7 \%)$ \\
STOP-Bang score 8 & $1(0.5 \%)$ & $0(0.0 \%)$ & $1(0.3 \%)$ \\
\hline
\end{tabular}

Notes: There were no statistically significant differences between the opioid user and non-user groups on the Epworth Sleepiness Scale ( $P=0.59)$ or for STOP-Bang positivity $(P=0.84)$. *Not all participants answered all questions.

with their health care provider. The implication is that many of these individuals may be undiagnosed and unrecognized, supported by the fact that fewer than a quarter of our population reported having been diagnosed and treated for sleep apnea by a physician. Although the screening questionnaires are not independently sufficient to make a clinical diagnosis, the discrepancy between the percentage screening at risk and the percentage reporting being diagnosed by a medical provider is striking. Given all of the health repercussions, such as cardiovascular and cerebrovascular disease, that come with a diagnosis of sleep apnea, special attention to the increased risk in the chronic pain population is warranted.
In recent years it has become common to treat chronic pain with opioids. However, opioid use is associated with substantial side effects and approximately $80 \%$ of individuals prescribed opioids will experience at least one side effect. ${ }^{25}$ In addition, patients using opioids may suffer respiratory depression, ${ }^{26}$ endocrine effects, addiction, and fatal overdose. Paradoxically, patients may experience opioid-induced hyperalgesia ${ }^{27}$ with worsening pain in response to certain types of pain stimuli.

Opioids inhibit the basic sleep-wake mechanisms by inhibiting transmission through central cholinergic and adenosinergic pathways. ${ }^{28}$ In addition to central apnea, opi- 


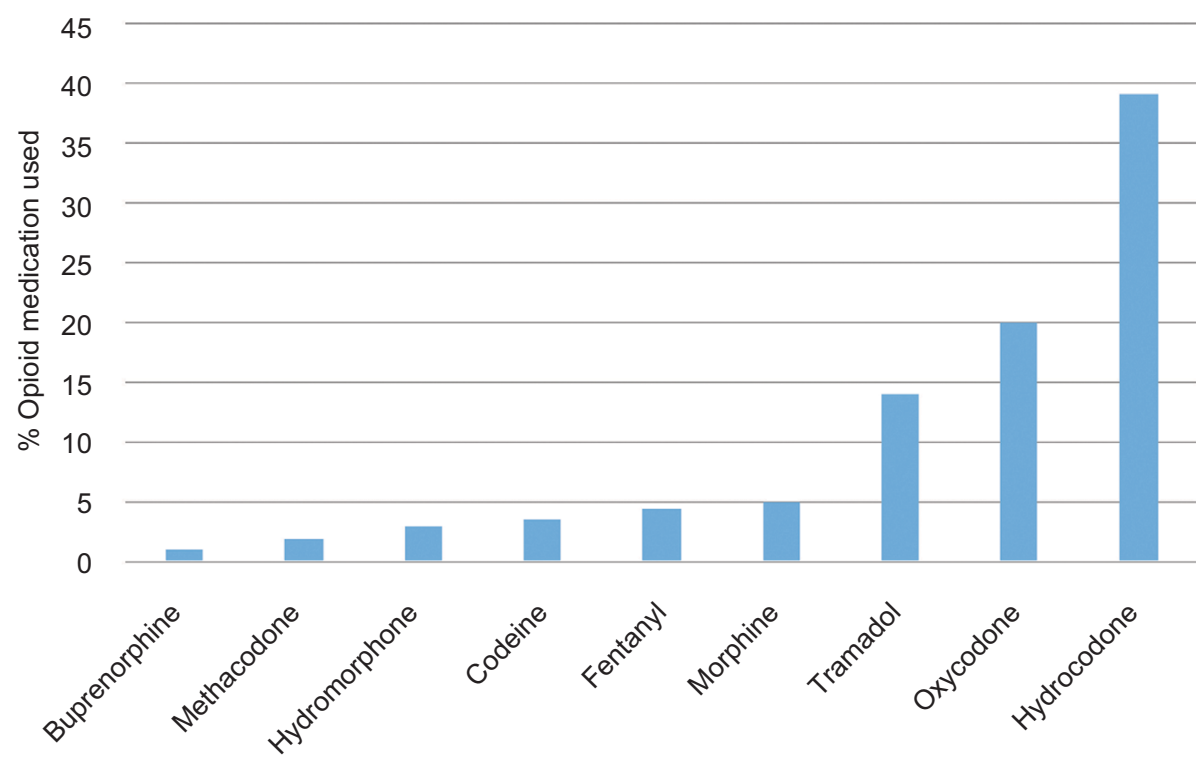

Opiate pain medications prescribed

Figure I Opiate drug distribution among subjects.

oids can contribute to OSA by deeply relaxing the tissues of the upper airway leading to mechanical obstruction. ${ }^{29}$ Sleepdisordered breathing may increase the sensitivity of both central and airway effects of opioids, while sleep disruption, intermittent hypoxemia, and systemic inflammation influence pain behavior and sensitivity to opioids. ${ }^{30}$

Given the association between opioid treatment and sleepdisordered breathing, it was expected that our CNCP patients using opioids would be at greater risk of sleep apnea. Against expectation, there was no difference in STOP-Bang positivity (score $\geq 3$ ) between opioid users and non-users. This prompts the question whether opioids really do increase the risk of OSA in chronic pain patients, and whether the STOP-Bang questionnaire is appropriate to identify an increased risk for sleep apnea, in particular OSA, in opioid users. The choice and dose of opioid medication were "given to effect". In hindsight, data collection of opioid dose as well as which medication, might have revealed a relationship between higher "morphine equivalent doses" and an increased frequency of sleep-disordered breathing. If such a relationship were to exist, then a case could be made for mandatory sleep-disordered breathing screening in all patients taking high morphine equivalent doses (for example, exceeding $200 \mathrm{mg} /$ day).

One possibility is that there may be co-occurrence of central apnea and obstructive apnea with opioid use. The questions on the STOP-Bang questionnaire is useful in screening for obstructive sleep apnea rather than central sleep apnea. Indeed, these factors may well be absent in a person experiencing CSA. Therefore, the STOP-Bang questionnaire may not be the best instrument to identify increased total apnea risk due to opioid therapy. However, daytime sleepiness which could be due to either central or peripheral apnea mechanisms, was the same in both opioid user and non-user patients. On the Epworth Sleepiness Scale, approximately $25 \%$ of both opioid users and non-users scored $>10$ associated with "excessive daytime sleepiness", ${ }^{24}$ perhaps suggesting that there is not an excess of central apnea in the opioid using patients.

If the measures contributing to the STOP-Bang score were more prevalent in the non-opioid group, this might have hidden the effect of opioids on apnea. However, patient demographics were extremely similar in both the opioid and non-opioid user groups. Age, gender, and BMI, all known to be associated with $\mathrm{OSA}^{2-4}$ and addressed by the questions in the Bang section of the questionnaire, were not different between opioid users and non-users (Table 1). There were no significant differences in any of these demographic parameters that might explain why the opioid user group did not have a higher proportion of patients with a positive STOP-Bang score.

Concomitant use of hypnotic medications such as benzodiazepines has been shown to worsen sleep apnea. They have the potential to cause complete OSA in heavy snorers or short repetitive CSAs. ${ }^{31}$ When used in conjunction with opioid medications these effects can be even more pronounced, making this a potentially lethal combination. In this survey, concomitant use of opioids and a hypnotic agent 
did not appear to increase the risk of OSA compared with use of opioids alone.

One possibility for the finding that the STOP-Bang questionnaire did not differentiate opioid users and non-users is that it did not distinguish the severity of apnea risk. In this study the STOP-Bang scores were dichotomized, by design, into positive or negative with a cut-off threshold score of $\geq 3$. However, increasing STOP-Bang scores reportedly predict the probabilities from none through moderate to severe OSA. ${ }^{19}$ In this study cohort, the demographic data showed that the risk of OSA is high with older, overweight patients already scoring high on the Bang section of the questionnaire. An analysis of the STOP-Bang scores at all levels, from 0 to 8 , showed no significant association with opioid use (Table 3), suggesting that the severity of OSA in opioid users is not greater than in opioid non-users.

Approximately one third of both the opioid-using and non-using patients had discussed the risk of sleep apnea with a health care provider. Fewer had been tested for sleep apnea. Overall, only approximately $20 \%$ of patients were diagnosed and treated for sleep apnea. These observations suggest a knowledge gap in patients being prescribed opioids when it comes to being warned of sleep-disordered breathing as a side effect. More reliable approaches can be designed to educate patients about the side effects of opioids, including sleep disorders and addiction, ${ }^{15,16}$ to ensure a true understanding of the risks and benefits of opioids. Previous studies have estimated the risk of sleep apnea in the chronic pain population using opioids to be as high as $75 \%$. The low percentages of patients in this survey tested and diagnosed may point to incomplete screening, testing, and diagnosis in this high-risk patient population. This large disparity between patients at risk and patients being diagnosed indicates that a very high percentage of opioid-using and non-using patients may be undiagnosed and untreated for sleep apnea.

We acknowledge several possible limitations to this study. The first limitation is that our study population was from an academic hospital and may not be representative of the pain population as a whole. Patients attending academic hospitals may be wealthier and better insured than the general pain population. Patients in this particular pain clinic were receiving consultation while prescribing was generally under the auspice of longitudinal providers such as the primary care clinicians. Another potential limitation of our study was the survey design. All information was anonymously self-reported by subjects and not confirmed by health records, which may lead to a self-report bias. Although the STOP-Bang OSA screening questionnaire has been validated in several populations, it has not been validated in the chronic pain population. This is a major limitation in that the STOP-Bang questionnaire, as generally applied, requires only three positive responses to define a high risk of OSA. In our population, many were older, overweight, and likely to have an increased neck circumference, reducing the sensitivity of the STOP-Bang score. However, even a comparison of STOP-Bang scores across the full range (from 0 to 8 ) did not reveal a difference in the frequency of OSA between opioid users and non-users. A clinician neither separately interviewed nor examined the subjects to confirm a diagnosis of sleep apnea, nor was polysomnography included as part of the study to validate the findings of the sleep apnea questionnaire. Although the STOP-Bang questionnaire has a relatively high sensitivity and specificity for the identification of OSA, polysomnography is the "gold standard" since the STOP-Bang questionnaire cannot identify other forms of sleep-disordered breathing such as CSA.

In conclusion, a large percentage of chronic pain subjects screened positive for OSA, regardless of opioid use. Many had never discussed their risk of sleep apnea with a health care professional nor were they personally aware that opioids may have increased their risk for sleep apnea. Given the estimated high prevalence of sleep apnea in patients on chronic opioids, our study indicates that a large proportion of our patients were undiagnosed and untreated, raising significant patient safety concerns. These findings suggest that physicians prescribing opioids need to be more conscious of the serious risks for sleep-disordered breathing leading to sleep apnea. There is a need for both greater awareness of both CSA and OSA in the chronic pain population and a proactive approach to provider discussion about sleep disorders. Education for prescribers, who are mostly in general medical practices, is greatly needed. Our initial study with the STOP-Bang questionnaire showed that it is simple to use and does identify patients at risk of sleep apnea. Future research is needed to validate a screening tool to properly identify those chronic pain patients at increased risk of sleep apnea, so that they can be appropriately tested with polysomnography and treated if their diagnosis is confirmed.

\section{Acknowledgments}

The project described was supported by the National Center for Advancing Translational Sciences (NCATS) and National Institutes of Health (NIH), through grant number UL1 TR000002. Thank you to Shannon Romero for helping to coordinate the distribution of surveys in a very busy clinic and to Maryam and Bushra Shaikh for being well-organized and great research assistants. Dr Ian Hutchinson assisted with editing and formatting this article. 


\section{Disclosure}

The authors report no conflicts of interest in this work.

\section{References}

1. Institute of Medicine of the National Academies Report. Relieving Pain in America: A Blueprint for Transforming Prevention, Care, Education, and Research. Washington DC: National Academies Press; 2011.

2. Myers KA, Mrkobrada M, Simel D. Does this patient have obstructive sleep apnea? The rational clinical examination systematic review. JAMA. 2013;317:731-741.

3. Qaseem A, Dallas P, Owens DK, et al. Diagnosis of obstructive sleep apnea in adults: a clinical practice guideline from theAmerican College of Physicians. Ann Intern Med. 2014;161(3):210-220.

4. Franklin KA, Lindberg E. Obstructive sleep apnea is a common disorder in the population- a review of the epidemiology of sleep apnea. J Thor Dis. 2015;7:1311-1322.

5. Pattinson KT. Opioids and the control of respiration. Br J Anaesth. 2008;100(6):747-758.

6. Victor LD. Obstructive sleep apnea. Am Fam Physician. 1999;60(8): 2279-2286.

7. Young T, Finn L, Peppard PE, et al. Sleep disordered breathing and mortality: eighteen-year follow-up of the Wisconsin sleep cohort. Sleep. 2008;31(8):1071-1078.

8. Webster LR, Choi Y, Desai H, Webster L, Grant BJ. Sleep-disordered breathing and chronic opioid therapy. Pain Med. 2008;9(4):425-432.

9. Onen SH, Onen F, Courpron P, Dubray C. How pain and analgesics disturb sleep. Clin J Pain. 2005;21(5):422-431.

10. Portenoy RK, Foley KM. Chronic use of opioid analgesics in nonmalignant pain: report of 38 cases. Pain. 1986;25(2):171-186.

11. Brennan F, Carr DB, Cousins M. Pain management: a fundamental human right. Anesth Analg. 2007;105(1):205-221.

12. Sullivan MD, Howe CQ. Opioid therapy for chronic pain in the United States: promises and perils. Pain. 2013;154 Suppl 1:S94-S100.

13. Hodge JG, Wetter SA, Noe SA. Emerging Legal Responses to Curb the Opioid Epidemic. The Journal of Law, Medicine \& Ethics. 2017;45(3):460-463.

14. Soelberg CD, Brown RE, du Vivier D, Meyer JE, Ramachandran BK. The US Opioid Crisis: Current Federal and State Legal Issues. Anesth Analg. 2017;125(5):1675-1681.

15. Volkow ND, Mclellan AT. Opioid Abuse in Chronic Pain--Misconceptions and Mitigation Strategies. N Engl J Med. 2016;374(13): $1253-1263$.
16. Ballantyne JC. Opioids for the Treatment of Chronic Pain: Mistakes Made, Lessons Learned, and Future Directions. Anesth Analg. 2017;125(5):1769-1778.

17. Walker JM, Farney RJ, Rhondeau SM, et al. Chronic opioid use is a risk factor for the development of central sleep apnea and ataxic breathing. J Clin Sleep Med. 2007;3(5):455-461.

18. Farney RJ, Walker JM, Cloward TV, Rhondeau S. Sleep-disordered breathing associated with long-term opioid therapy. Chest. 2003;123(2):632-639.

19. Chung F, Abdullah HR, Liao P, Questionnaire S-B. STOP-Bang Questionnaire: A Practical Approach to Screen for Obstructive Sleep Apnea. Chest. 2016;149(3):631-638.

20. Abrishami A, Khajehdehi A, Chung F. A systematic review of screening questionnaires for obstructive sleep apnea. Can J Anaesth. 2010;57(5):423-438.

21. Vasu TS, Doghramji K, Cavallazzi R, et al. Obstructive sleep apnea syndrome and postoperative complications: clinical use of the STOPBANG questionnaire. Arch Otolaryngol Head Neck Surg. 2010;136(10): 1020-1024.

22. Kumar S, Mcelligott D, Goyal A, Baugh M, Ionita RN. Risk of Obstructive Sleep Apnea (OSA) in Hospitalized Patients. Chest. 2010;138(4): 779A.

23. Ong TH, Raudha S, Fook-Chong S, Lew N, Hsu AA, Aal H. Simplifying STOP-BANG: use of a simple questionnaire to screen for OSA in an Asian population. Sleep Breath. 2010;14(4):371-376.

24. Johns MW. A new method for measuring daytime sleepiness: the Epworth sleepiness scale. Sleep. 1991;14(6):540-545.

25. Furlan AD, Sandoval JA, Mailis-Gagnon A, Tunks E. Opioids for chronic noncancer pain: a meta-analysis of effectiveness and side effects. Can Med Assoc J. 2006;174(11):1589-1594.

26. Jungquist CR, Flannery M, Perlis ML, Grace JT. Relationship of chronic pain and opioid use with respiratory disturbance during sleep. Pain Manag Nurs. 2012;13(2):70-79.

27. Lee M, Silverman SM, Hansen H, Patel VB, Manchikanti L. A comprehensive review of opioid-induced hyperalgesia. Pain Physician. 2011;14(2):145-161.

28. Lam KK, Kunder S, Wong J, Doufas AG, Chung F. Chronic sleep apnea, pain, and opioids: is the riddle solved? Chest. 2016;29:134-140.

29. Hudgel DW, Martin RJ, Johnson B, Hill P. Mechanics of the respiratory system and breathing pattern during sleep in normal humans. $J$ Appl Physiol Respir Environ Exerc Physiol. 1984;56(1):133-137.

30. Doufas AG. Obstructive Sleep Apnea, Pain, and Opioid Analgesia in the Postoperative Patient. Curr Anesthesiol Rep. 2014;4(1):1-9.

31. Guilleminault C. Benzodiazepines, breathing, and sleep. Am J Med. 1990;88(3A):25S-28S.
Nature and Science of Sleep

\section{Publish your work in this journal}

Nature and Science of Sleep is an international, peer-reviewed, open access journal covering all aspects of sleep science and sleep medicine, including the neurophysiology and functions of sleep, the genetics of sleep, sleep and society, biological rhythms, dreaming, sleep disorders and therapy, and strategies to optimize healthy sleep. The manuscript

\section{Dovepress}

management system is completely online and includes a very quick and fair peer-review system, which is all easy to use. Visit http://www. dovepress.com/testimonials.php to read real quotes from published authors. 